\title{
Making Sense of Gray Matter Abnormalities in Chronic Orofacial Pain-Synthesizing Divergent Findings
}

\author{
Massieh Moayedi, ${ }^{1,2}$ Danielle DeSouza, ${ }^{1,2}$ and Nathalie Erpelding ${ }^{2}$ \\ ${ }^{1}$ Institute of Medical Science, University of Toronto, Toronto, Ontario M5S 1A8, Canada, ${ }^{2}$ Division of Brain, Imaging and Behaviour-Systems Neuroscience, \\ Toronto Western Research Institute, University Health Network, Toronto, Ontario M5T 2S8, Canada \\ Review of Gustin et al.
}

Several studies have investigated various chronic pain populations for structural brain abnormalities in gray matter (GM). Most studies report decreases in GM volume and/or thickness in regions related to antinociceptive, cognitive, and/or limbic functions (May, 2011). Conversely, some of these studies have identified GM increases in nociceptive regions (e.g., Younger et al., 2010; Moayedi et al., 2011). Although there is some level of convergence between these findings, it is likely that some GM changes are specific to particular chronic pain populations and may be attributed to differences in etiology and symptomology. For instance, patients with trigeminal neuralgia (TN) report tingling and sharp shocklike symptoms in the affected region of the face, whereas patients with temporomandibular disorder (TMD) report deep, aching pain. Elucidating brain changes specific to these trigeminal pain conditions may provide insight into novel treatment approaches more specific to TN or TMD.

A recent study by Gustin et al. (2011) compared GM abnormalities in individuals with different chronic facial pain conditions: TMD and trigeminal neuropathic

Received June 17, 2011; revised July 20, 2011; accepted July 20, 2011.

The authors thank Drs. Karen Davis, Jarred Younger, and Tim Salomons for feedback on this Journal Club.

Correspondence should be addressed to Massieh Moayedi, Division of Brain, Imaging and Behaviour-Systems Neuroscience, Toronto Western Research Institute, University Health Network, Toronto Western Hospital, MP14-301, 399 Bathurst Street, Toronto, ON M5T 258, Canada. E-mail: mmoayedi@uhnres.utoronto.ca.

DOI:10.1523/JNEUROSCI.3103-11.2011

Copyright $\odot 2011$ the authors $\quad 0270-6474 / 11 / 3112396-02 \$ 15.00 / 0$ pain (TNP), which included patients with $\mathrm{TN}$ and trigeminal neuropathy (TNeu). The authors describe that these patient groups differ in etiology as TNP is caused by direct nerve damage, whereas TMD is a musculoskeletal chronic pain disorder associated with increased nociceptive input into the brain. The authors hypothesized that TNP, but not TMD, is associated with reduced thalamic volume, as well as abnormal thalamic biochemistry.

A particular strength of this study is that the authors used a combination of structural and functional magnetic resonance imaging (MRI) and magnetic resonance spectroscopy (MRS) to examine structure and neuronal viability in the thalamus simultaneously. Together, this multimodal approach provides a deeper understanding of changes in GM volume. Functional MRI (fMRI) was used to locate the thalamic region of interest: the sensory thalamic nucleus and MRS was used to evaluate the $N$-acetylaspartate/creatine ratio (NAA:Cr); a marker of cell metabolism in neurons and oligodendrocytes. Studies in stroke, ischemia, and other neurodegenerative processes have found that NAA:Cr predicts disease outcome, and may be a marker of neuronal viability (Moffett et al., 2007). Gustin et al. found decreased GM volume and NAA:Cr in the thalamus of the TNP group (an effect driven by the TNeu patients). This suggests that GM volumetric decreases are related to reduced neuronal viability. This finding is interesting in light of recent studies (e.g., Seminowicz et al., 2011), which suggest that GM decreases are sometimes reversed after the source of pain has been resolved. Evidence from animal studies indicates that remodeling of neuronal processes (measured by Growth Associated Protein-43; GAP-43) may be responsible for these MRI-detectable morphological brain changes, as higher levels of GAP-43 correlated with larger structural volumes (Lerch et al., 2011). However, reduced metabolism does not equate cell death and neurons can recover. Therefore, MRS could potentially elucidate whether the reversal of MRI-detectable GM changes is related to increased neural viability. Future work is required to determine the histological correlates of structural plasticity in chronic pain and how these processes may change once pain is resolved. It is possible that different chronic facial pain disorders affect neuronal viability differently because of differences in symptom etiology (i.e., nerve trauma vs constant nociceptor activation).

Gustin and colleagues' study supports their hypothesis that TMD patients have a different pattern of GM abnormality than TNP patients. Specifically, the TMD group showed no structural GM change, whereas the TNP group showed widespread decreases in GM volume in regions involved in nociceptive processing, pain modulation, and motor response, compared to healthy, pain-free controls. The findings in the TMD group are puzzling, however, because several previous studies 
identified significant GM differences in TMD patients (Younger et al., 2010; Gerstner et al., 2011; Moayedi et al., 2011). This discrepancy might have resulted from differences in the type of TMD in the populations studied. TMDs are a group of disorders that affect the temporomandibular joint and the muscles of mastication, with a heterogeneous population of patients. Some TMD patients have functional and idiopathic pain, whereas other patients, specifically those who have suffered some form of trauma, show a clear peripheral disruption (e.g., disc derangement in the TM joint). To address issues arising from this heterogeneity, Dworkin and LeResche (1992) established the TMDResearch diagnostic criteria. Previous studies examining GM abnormalities in TMD have provided inclusion and exclusion criteria for TMD patients, for example, restricting recruitment to the myofascial TMD subtype (Younger et al., 2010; Gerstner et al., 2011), or to patients with idiopathic (non-traumatic) TMD (Moayedi et al., 2011). In the paper by Gustin et al., specific diagnostic criteria to identify patients with TMD were not included. Hence, the potential heterogeneity of TMD patients participating in the Gustin et al. study may account for the lack of group differences between patients with TMD and controls.

As previously mentioned, patients in the TNP group were categorized as having either TNeu or TN, based on the Liverpool criteria (Nurmikko and Eldridge, 2001). According to these criteria, TNeu is characterized by dull or sharp enduring pain with shooting pain superimposed, whereas typical TN is described as sharp, shooting pain lasting seconds to minutes. These different symptoms may result from unique etiologies of these facial pain disorders: TNeu is thought to be caused by direct trauma to the trigeminal nerve, whereas TN is associated with neurovascular compression, tumor growth, or demyelination. More recent classification systems have been proposed to further distinguish between different types of facial pain. For example, TN secondary to multiple sclerosis (MS) or tumor growth constitutes a separate group from idiopathic TN, often the result of neurovascular compression (Eller et al., 2005). The reason for this shift in classification is that patient symptomology can be highly variable between patients with idiopathic TN and patients with facial pain secondary to MS or tumor growth. Gustin et al. did not provide information regarding the etiology of patient pain for either TNP subgroup. Future studies may consider incorporating the new facial pain classification systems into screening to better characterize group patients according to etiology and symptomology.

Although Gustin et al. used a multimodal approach to test their hypotheses, there are some design considerations that should be taken into account in future studies. For example, to better localize the ventral posteromedial nucleus of the thalamus (involved in sensory processing of the face), this study used a functional localizer, which consisted of innocuous brushing of the bottom lip on the right side of the face. The authors determined that this thalamic activation overlapped with the area of GM loss in the TNP group, driven by the TNeu subgroup. Although the authors carefully divided patients into TN and TNeu groups, they did not control for the subjects' pain site (left, right, or bilateral). Becerra et al. (2006) reported that $\mathrm{TN}$ patients had different patterns of brain activation, including the thalamus, in response to pain evoked by innocuous brushing of the affected and unaffected sides of the neuropathic pain. Therefore, the functional localization technique used in the present study should be interpreted with caution.

One of the difficulties of studying a unilateral pain syndrome is comparing the brains of patients with pain on different sides of the body. Gustin et al. (2011) reflect the brain images of six TN patients with unilateral left-sided facial pain with respect to the $X$-plane. Although this step artificially created a more homogenous right-sided group of pain patients, it prevents interpretation with regard to the laterality of the findings. One method to remove this potential confound in future studies may be to include separate groups of patients with left pain and right pain and investigate potential lateralized GM volume abnormalities.

In conclusion, the study by Gustin and colleagues provides relevant insights into GM abnormalities in chronic facial pain. This study relates MRI-detectable GM abnormalities to a molecular marker to measure neuronal viability in chronic facial pain patients. Additionally, the authors report that reduced GM in the thalamus is related to decreased neuronal viability in TNP, but not in TMD. Nonetheless, as highlighted in this commentary, some of the findings may be attributable to patient classification and to the use of methodological approaches. Future studies should take these considerations into account to most accurately study GM differences in different chronic pain conditions.

\section{References}

Becerra L, Morris S, Bazes S, Gostic R, Sherman S, Gostic J, Pendse G, Moulton E, Scrivani S, Keith D, Chizh B, Borsook D (2006) Trigeminal neuropathic pain alters responses in CNS circuits to mechanical (brush) and thermal (cold and heat) stimuli. J Neurosci 26: 10646-10657.

Dworkin SF, LeResche L (1992) Research diagnostic criteria for temporomandibular disorders: review, criteria, examinations and specifications, critique. J Craniomandib Disord 6:301355.

Eller JL, Raslan AM, Burchiel KJ (2005) Trigeminal neuralgia: definition and classification. Neurosurg Focus 18:E3.

Gerstner G, Ichesco E, Quintero A, SchmidtWilcke $T$ (2011) Changes in regional gray matter and white matter volume in patients with myofascial-type temporomandibular disorders: a voxel-based morphometry study. J Orofac Pain 25:99-106.

Gustin SM, Peck CC, Wilcox SL, Nash PG, Murray GM, Henderson LA (2011) Different pain, different brain: thalamic anatomy in neuropathic and non-neuropathic chronic pain syndromes. J Neurosci 31:5956-5964.

Lerch JP, Yiu AP, Martinez-Canabal A, Pekar T, Bohbot VD, Frankland PW, Henkelman RM, Josselyn SA, Sled JG (2011) Maze training in mice induces MRI-detectable brain shape changes specific to the type of learning. Neuroimage 54:2086-2095

May A (2011) Structural brain imaging: a window into chronic pain. Neuroscientist 17: 209-220.

Moayedi M, Weissman-Fogel I, Crawley AP, Goldberg MB, Freeman BV, Tenenbaum HC, Davis KD (2011) Contribution of chronic pain and neuroticism to abnormal forebrain gray matter in patients with temporomandibular disorder. Neuroimage 55:277-286.

Moffett JR, Ross B, Arun P, Madhavarao CN, Namboodiri AMA (2007) N-Acetylaspartate in the CNS: from neurodiagnostics to neurobiology. Prog Neurobiol 81:89-131.

Nurmikko TJ, Eldridge PR (2001) Trigeminal neuralgia-pathophysiology, diagnosis and current treatment. Br J Anaesth 87:117-132.

Seminowicz DA, Wideman TH, Naso L, HatamiKhoroushahi Z, Fallatah S, Ware MA, Jarzem P, Bushnell MC, Shir Y, Ouellet JA, Stone LS (2011) Effective treatment of chronic low back pain in humans reverses abnormal brain anatomy and function. J Neurosci 31:75407550

Younger JW, Shen YF, Goddard G, Mackey SC (2010) Chronic myofascial temporomandibular pain is associated with neural abnormalities in the trigeminal and limbic systems. Pain 149:222-228. 